\title{
A STATISTICAL APPROACH TO ANALYSE EFFECT OF MISALIGNMENTS AND CORRECTION ALGORITHMS IN HIGH-ENERGY LINACS
}

\author{
S. Fartoukh, CERN, 1211-Geneva 23, Switzerland
}

\section{Abstract}

This paper presents a new and general tracking method capable of analysing, in a statistical way, the dipole wakefield effects on a high-energy charged-particle beam.

\section{EQUATION OF MOTION}

We consider an electron bunch travelling along a line containing magnetic quadrupoles and RF structures. We note $s$ the longitudinal coordinate along the line, and $z$, a relative position within the bunch ( $z>0$ at the bunch tail). We will assume the beam to be fully relativistic so that the longitudinal distribution of particles $\rho(z)$ is completely rigid along the $\hat{s}$ axis. For a slice of charge at a relative position $z$ inside the bunch, we note $\delta x(s, z)$ the transverse position (horizontal or vertical) of its centre of gravity, $x(s, z)=\delta x(s, z)+\tilde{x}(s, z)$ the position of any particle within the slice, and $\gamma(s, z) m_{e} c^{2}$ its energy (thus, $\tilde{x}$ describes the betatron motion in the presence of acceleration). Then, the fundamental dynamics relation, projected on the transverse and longitudinal planes, gives [1]:

$$
\begin{gathered}
\left\{\begin{array}{l}
x^{\prime \prime}(s, z)+\gamma^{\prime}(s, z) / \gamma(s, z) x^{\prime}(s, z)+k(s, z) x(s, z) \\
=\frac{e^{2} / m_{e} c^{2}}{\gamma(s, z)} \int_{-\infty}^{z} W_{T}^{\delta}\left(z-z^{*}\right) \rho\left(z^{*}\right) \delta x\left(z^{*}\right) d z^{*}
\end{array}\right. \\
\gamma^{\prime}(s, z)=e / m_{e} c^{2}\left(G_{R F}(s, z)-e \int_{-\infty}^{z} \rho\left(z^{*}\right) W_{L}^{\delta}\left(z-z^{*}\right) d z^{*}\right)
\end{gathered}
$$

Here, $W_{L}^{\delta}$ and $W_{T}^{\delta}$ are the longitudinal and transverse delta-function wake potentials, $k(s, z)\left[\mathrm{m}^{-2}\right]$ is the quadrupole strength seen by the slice $z$ and $G_{R F}(s, z)[\mathrm{V} / \mathrm{m}]$ represents the accelerating field along the line ${ }^{1}$. Summing Eq. 1 over all the particles of the slice $z$, we obtain the equations verified by $\delta x(s, z)$ and $\tilde{x}(s, z)$ :

$$
\begin{aligned}
& \left\{\begin{array}{l}
\delta x^{\prime \prime}(s, z)+\gamma^{\prime}(s, z) / \gamma(s, z) \delta x^{\prime}(s, z)+k(s, z) \delta x(s, z) \\
=\frac{e^{2} / m_{e} c^{2}}{\gamma(s, z)} \int_{-\infty}^{z} W_{T}^{\delta}\left(z-z^{*}\right) \rho\left(z^{*}\right) \delta x\left(z^{*}\right) d z^{*}
\end{array}\right. \\
& \tilde{x}^{\prime \prime}(s, z)+\gamma^{\prime}(s, z) / \gamma(s, z) \tilde{x}^{\prime}(s, z)+k(s, z) \tilde{x}(s, z)=0
\end{aligned}
$$

Eq. 3 is the the well-known Hill equation and the classical theory of the beam matrix and $R$ matrix, not reported here, can be applied in order to track the " $\tilde{x}$-envelope" of each slice all along the line ${ }^{2}$. Eq. 2 has no analytical solution in the most general case and its numerical treatment is presented hereafter.

\footnotetext{
${ }^{1} G_{R F}(s, z)=0$ and $k(s, z)=G_{q}(s) e /(\gamma(s, z) m c)$ in quadrupoles, $G_{q}(s)[\mathrm{T} / \mathrm{m}]$ being the step function which describes the quadrupole gradient within the line; $G_{R F}(s, z)=G_{R F} \cos \left(\omega_{R F} z / c-\phi_{R F}\right)$ and $k(s, z)=0$ in RF structures.

${ }^{2}$ In this model, we see that the motion of $\tilde{x}$ is totally independent of the one of $\delta x$; this would not be the case anymore if, for instance, we had taken into account the quadrupole mode of the wakes.
}

\section{GENERALISED R-MATRIX}

By splitting the bunch into $n_{s}$ slices of thickness $\triangle z$, we can write the integro-differential equation 2 as a vectorial differential equation of order one and dimension $2 n_{s}$ :

$$
\mathcal{X}^{\prime}(s)=\mathcal{A}(s) * \mathcal{X}(s)
$$

where $\mathcal{X}(s)=\left[\delta x\left(s, z_{1}\right), \delta x^{\prime}\left(s, z_{1}\right) \ldots \delta x\left(s, z_{n_{s}}\right), \delta x^{\prime}\left(s, z_{n_{s}}\right)\right]$ and where $\mathcal{A}$ is the following $2 n_{s} \times 2 n_{s}$ matrix:

$$
\mathcal{A}=\left(\begin{array}{cccc}
A_{1}^{0} & 0 & \ldots & 0 \\
A_{21} & A_{2}^{0} & \ldots & 0 \\
\vdots & \vdots & \ddots & \vdots \\
A_{n_{s} 1} & A_{n_{s} 2} & \ldots & A_{n_{s}}^{0}
\end{array}\right) \text { with }\left\{\begin{array}{c}
A_{i}^{0}=\left(\begin{array}{cc}
0 & 1 \\
-k\left(s, z_{i}\right)-\frac{\gamma^{\prime}\left(s, z_{i}\right)}{\gamma\left(s, z_{i}\right)}
\end{array}\right) \\
A_{i j}=\left(\begin{array}{cc}
0 & 0 \\
\triangle z \rho\left(z_{j}\right) W_{T}^{\delta}\left(z_{i}-z_{j}\right) & 0
\end{array}\right) .
\end{array}\right.
$$

The solution of the previous equation can be written as $\mathcal{X}(s)=\mathcal{R}(s) * \mathcal{X}(0)$, where $\mathcal{R}(s)$ is a $2 n_{s} \times 2 n_{s}$ matrix that we call "generalised R-matrix in the presence of wakefield" and which verifies the matricial differential equation:

$$
\mathcal{R}^{\prime}(s)=\mathcal{A}(s) * \mathcal{R}(s) \text { with } \mathcal{R}(0)=\mathbf{I d}
$$

We can easily see that, like the $\mathcal{A}$ matrix, $\mathcal{R}$ is a lower triangular $2 \times 2$ block matrix: the diagonal blocks $R_{i i}^{0}$ describe the motion of the slices $z_{i}$ without any wakefields $\left(R_{i i}^{0^{\prime}} \stackrel{5}{=} A_{i}^{0} * R_{i i}^{0}\right)$ whereas the lower blocks $R_{i j}, i>j$, testify to the interaction between the slices $z_{i}$ and $z_{j}$ due to the transverse wake. Certainly more complicated than a classical tracking, this method has nevertheless the advantage of a very practical computation of the beam line sensitivity to the displacements of its components (next section).

\section{BEAM LINE SENSITIVITY}

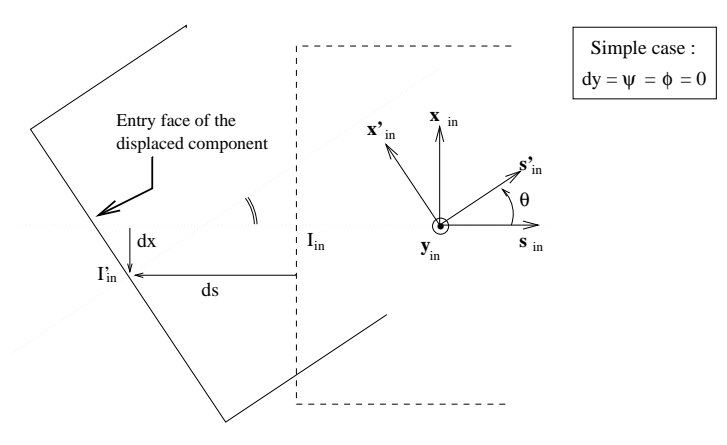

Figure 1:

Here, we are interested in the effects of transverse misalignments of the beam line components. Assuming the elements to be totally rigid objects, their position along the 
line can be defined by six parameters (see Fig. 1); first, we execute a global translation of the component, then, three rotations, leaving invariant the middle point of the displaced entry face of the component ( $I_{\text {in }}^{\prime}$ in Fig. 1):

- the horizontal, vertical and longitudinal displacements $d x, d y$ and and $d s$ respectively.

- the rotation $\theta$ around the actual vertical axis, $\hat{y}_{\text {in }}$ in Fig. 1, or yaw angle, the rotation $\phi$ around the newly defined horizontal axis, $\hat{x}_{\text {in }}^{\prime}$ in Fig. 1, or pitch angle, the rotation $\psi$ around the newly defined longitudinal axis or roll angle.

We will forget here the "longitudinal alignment errors", $d s$ and $\psi$, which do not create any transverse offset ${ }^{3}$. Then, we consider a component of length $L$ with the misalignments $d x$ and $\theta$, and we suppose that the beam goes into the component (abscissa $s_{0}$ ) with the initial conditions $\mathcal{X}\left(s_{0}\right)$; using the previous section, we can compute the beam offset at the component exit $\left(s_{1}=s_{0}+L\right)$ in the following way:

$$
\mathcal{X}\left(s_{1}\right)=\mathcal{R}\left(s_{0} \rightarrow s_{1}\right)\left(\mathcal{X}\left(s_{1}\right)+\mathcal{X}_{\text {in }}(d x, \theta)\right)+\mathcal{X}_{\text {out }}(d x, \theta)
$$

where $\mathcal{X}_{\text {in }}(d x, \theta)=-[d x, \theta, \ldots, d x, \theta]$ and $\mathcal{X}_{\text {out }}(d x, \theta)=$ $[d x+L \theta, \theta, \ldots, d x+L \theta, \theta]$ refers to the change of coordinate at the component entry and at the component exit respectively. Thus, at any abscissa $s_{f}$, we can estimate the line sensitivity relative to the misalignments of any of its components; for instance, for a yaw angle $\theta_{i}$ of the element number $i$, we obtain:

$\partial_{\theta_{i}} \mathcal{X}\left(s_{f}\right)=\mathcal{R}\left(s_{i}+L_{i} \rightarrow s_{f}\right)\left(\Theta_{\text {out }}\left(L_{i}\right)+\mathcal{R}\left(s_{i} \rightarrow s_{i}+L_{i}\right) \Theta_{\text {in }}\right)$

with $\Theta_{\text {in }}=-[0,1 \ldots 0,1]$ and $\Theta_{\text {out }}\left(L_{i}\right)=\left[L_{i}, 1 \ldots L_{i}, 1\right]$. Finally, in the same way, we can obtain all the response coefficients of the line, concerning the initial offset (angle and position) of the bunch.

\section{CORRECTION ALGORITHMS}

The simplest alignment methods are based on a correction of the bunch centre-of-charge deviation measured at one or several beam position monitors (BPM) within the line by moving the quadrupoles transversely. More recently, new correction schemes have been proposed, based on the simultaneous minimisation of trajectory deviations and trajectory differences linked to variable quadrupole settings or linked to variable bunch charge or bunch length [2]. However that may be, all these correction techniques have in common the following fact: they all come down to the minimisation by moving quadrupoles of a certain function $\Phi$ which depends quadratically on measurements of trajectory deviations. Then, it can be shown [3] that after this minimisation the vector $\mathcal{X}\left(s_{f}\right)$ (containing the positions and angles of the $n_{s}$ slices at the line output) does not depend anymore on the quad misalignments before correction but

\footnotetext{
${ }^{3}$ For flat beams, the roll angle $\psi$, coupling both transverse planes, may become critical for the emittance growth in the plane where the beam dimension is the smallest, but, actually, it is not yet implemented in the method.
}

only and in a linear way on the errors of the line ${ }^{4}$, that we write in the formal following way:

$$
\mathcal{X}\left(s_{f}\right) \stackrel{\text { def }}{=} M_{e r} d X_{e r}
$$

where $d X_{e r}$ is a stochastic vector containing the "errors of the line" and where $M_{e r}$ is a $2 n_{s} \times N_{e r}$ matrix which depends on the focusing lattice (via the response coefficients) and on the choice of the correction algorithm (via the choice of the function $\Phi$ ).

\section{STATISTICAL BEAM RESPONSE IN TERM OF EMITTANCE GROWTH}

From now on the rest of the paper, the notation $\langle\mathcal{Q}\rangle_{e r}$ will represent the average of the quantity $\mathcal{Q}$ over the statistic distribution of the "errors", compared to the same notation, without suffix, referring to an average over the longitudinal particle distribution $\rho(z)$. So, we suppose that the vector, $\mathbf{m}_{\mathrm{er}} \equiv<d X_{e r}>_{e r}$, and the covariance matrix, $\Gamma_{e r} \equiv<$ $d X_{e r}{ }^{\mathrm{T}} d X_{e r}>_{e r}-\mathbf{m}_{\text {er }} \mathbf{m}_{\mathbf{e r}}$, are known quantities which depends on the pre-alignment scheme ${ }^{5}$.

For the sake of simplification, we will assume $\mathbf{m}_{\mathbf{e r}}=0$. By using Eq. 6, we can compute the average and the covariance matrix of the vector $\mathcal{X}\left(s_{f}\right)$ at the line output:

$$
\begin{aligned}
& \widetilde{\mathbf{m}} \stackrel{\text { def }}{=}<\mathcal{X}\left(s_{f}\right)>_{e r} \quad=M_{e r} \mathbf{m}_{\text {er }}=0 \\
& \widetilde{\Gamma} \stackrel{\text { def }}{=}<\mathcal{X}\left(s_{f}\right)^{\mathrm{T}} \mathcal{X}\left(s_{f}\right)>_{e r}=M_{e r} \Gamma_{e r}{ }^{\mathrm{T}} M_{e r}
\end{aligned}
$$

Nevertheless, for reasons which will appear later, we prefer to define the symmetric matrix $\Gamma_{1} \leq i \leq 2 n_{s}$ by:

$\left\{\begin{array}{l}\Gamma_{2 i-1,2 j-1}=\Delta Q_{i j}\left\langle\left(\delta x\left(z_{i}\right)-<\delta x>\right)\left(\delta x\left(z_{j}\right)-<\delta x>\right)\right\rangle_{e r} \\ \Gamma_{2 i, 2 j}=\triangle Q_{i j}\left\langle\left(\delta x^{\prime}\left(z_{i}\right)-<\delta x^{\prime}>\right)\left(\delta x^{\prime}\left(z_{j}\right)-<\delta x^{\prime}>\right)\right\rangle_{e r} \\ \Gamma_{2 i-1,2 j}=\Delta Q_{i j}\left\langle\left(\delta x\left(z_{i}\right)-<\delta x>\right)\left(\delta x^{\prime}\left(z_{j}\right)-<\delta x^{\prime}>\right)\right\rangle_{e r} \\ 1 \leq i \leq n_{s}, 1 \leq j \leq n_{s}, \text { where } \triangle Q_{i j} \stackrel{\text { def }}{=} \triangle z \sqrt{\rho\left(z_{i}\right) \rho\left(z_{j}\right)} \\ <\delta x>=\sum_{n=1}^{n_{s}} \triangle z \rho\left(z_{n}\right) \delta x\left(z_{n}\right) \text { and }<\delta x^{\prime}>=\sum_{n=1}^{n s} \triangle z \rho\left(z_{n}\right) \delta x^{\prime}\left(z_{n}\right)\end{array}\right.$

which can be easily computed from $\widetilde{\Gamma}$ and $\rho(z)$.

The beam matrix of the whole bunch at the line output is then given by: $\mathcal{S}=\left(\begin{array}{cc}\left.<x^{2}\right\rangle & <x x^{\prime}> \\ \left\langle x x^{\prime}\right\rangle & \left\langle x^{\prime 2}\right\rangle\end{array}\right)$ where the notations used are explained hereafter.

- $\left\langle x^{2}\right\rangle$ is the RMS value of the transverse beam extension at the line output. Since the motions of $\tilde{x}(z)$ and $\delta x(z)$ are uncorrelated (Section 1), we have:

$$
<x^{2}>=\int d z \rho(z)\left[\left(\sigma_{x}^{2}(z)+(\delta x(z)-<\delta x>)^{2}\right]\right.
$$

\footnotetext{
${ }^{4}$ From now on the rest of the paper, by "errors of the line", we will always refer to the structure and BPM misalignments, to the BPM and correction resolution, and to the initial conditions of the different trajectories considered in the correction algorithm.

${ }^{5}$ Here, we will only consider static misalignments of the structures, resulting from the linac pre-alignment strategy; then, we suppose that the characteristic time required to apply the correction remains very small compared to the characteristic period of the machine vibrations (ground motion).
} 
where $\sigma_{x}(z)$ represents the transverse size (RMS value) of the $z$-slice at the line output.

- In a similar way, and with evident notations, we have:

$<x^{\prime 2}>=\int d z \rho(z)\left[\sigma_{x^{\prime}}^{2}(z)+\left(\delta x^{\prime}(z)-<\delta x^{\prime}>\right)^{2}\right]$

$<x x^{\prime}>=\int d z \rho(z)\left[\sigma_{x x^{\prime}}(z)+(\delta x(z)-<\delta x>)\left(\delta x^{\prime}(z)-<\delta x^{\prime}>\right)\right]$

Thus, we can write the matrix $\mathcal{S}$ in the following manner:

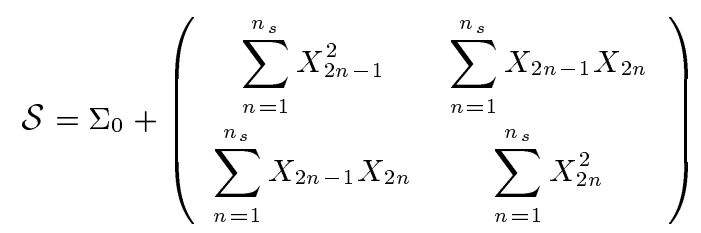

where $\Sigma_{0}$ is the beam matrix at the line output, assuming no error within the line ${ }^{6}$, and where $X$ is the $2 n_{s^{-}}$ dimensional vector defined by:

$$
\left\{\begin{aligned}
X_{2 n-1}=\sqrt{\triangle z \rho\left(z_{n}\right)}\left(\delta x\left(z_{n}\right)-<\delta x>\right), & & 1 \leq n \leq n_{s} \\
X_{2 n}=\sqrt{\triangle z \rho\left(z_{n}\right)}\left(\delta x^{\prime}\left(z_{n}\right)-<\delta x^{\prime}>\right), & & 1 \leq n \leq n_{s}
\end{aligned}\right.
$$

the covariance matrix of which is the matrix $\Gamma$ previously defined. Finally, the RMS emittance is defined as usual by:

$$
\epsilon(X) \stackrel{\operatorname{def}}{=} \sqrt{\operatorname{det}(\mathcal{S})}
$$

In order to go further in the computations, we are forced to make the following assumption: we assume the vector $X_{e r}$ to be Gaussian, so that the vector $X$ becomes also Gaus$\operatorname{sian}^{7}$. Hence, the knowledge of $\Gamma$ permits the complete description of the statistical distribution $\rho_{X}$ related to the vector $X$ :

$$
\rho_{X}(X)=\frac{1}{(2 \pi)^{n_{s}} \sqrt{\operatorname{det}(\Gamma)}} \exp \left(-\frac{1}{2} \mathrm{~T} \Gamma^{-1} X\right)
$$

The first and second moment of the emittance distribution are then:

$$
\begin{aligned}
<\epsilon>_{e r} & =\int_{\mathbf{R}^{2 n_{s}}} \epsilon(X) \rho_{X}(X) d X \\
<\epsilon^{2}>_{e r} & =\int_{\mathbf{R}^{2 n_{s}}} \epsilon^{2}(X) \rho_{X}(X) d X
\end{aligned}
$$

The second moment is easy to compute, since the function $\epsilon^{2}(X)$ is a polynomial in $X$ of degree 4 . For the computation of the first moment, a direct estimation by numerical integration is of course out of question (the computation time growing exponentially with $2 n_{s}$ ). In fact, we are able to reduce this integral to a 2 -dimensional integral (independently of $n_{s}$ ) and, under these conditions, we can compute

${ }^{6} \Sigma_{0}=\sum_{n=1}^{n_{s}} \rho\left(z_{n}\right) \triangle z R_{n, n}^{0} \Sigma_{i n}\left(z_{n}\right)^{\mathrm{T}} R_{n, n}^{0}$ where $\Sigma_{i n}\left(z_{n}\right)$ is the beam matrix of the slice $z_{n}$ at the line input and $R_{n, n}^{0}$, the diagonal block $(n, n)$ of the generalised R-matrix of the whole line, which is also the classical $2 \times 2$ transfer matrix describing the motion of the slice $z_{n}$ without any wakefield (Section 1).

${ }^{7} X$ depends linearly on the vector $\mathcal{X}$, Eq. 9 , which depends linearly on the vector $X_{e r}$, Eq. 6 . it numerically [3].

If now, we note $N(\epsilon)$, the percentage of machines which give, a final single-bunch emittance lower than $\epsilon$, we have:

$$
N(\epsilon)=\int_{\epsilon(X) \leq \epsilon} \rho_{X}(X) d X=\int_{\mathbf{R}^{2 n_{s}}} \Theta(\epsilon-\epsilon(X)) \rho_{X}(X) d X
$$

where $\Theta$ represents the Heaviside step function. Thus, by definition of the density $\rho_{\epsilon}$, we have:

$$
\rho_{\epsilon}(\epsilon) \stackrel{\text { def }}{=} \frac{d N}{d \epsilon}(\epsilon)=\int_{\mathbf{R}^{2 n_{s}}} \delta(\epsilon-\epsilon(X)) \rho_{X}(X) d X
$$

where $\delta$ is the Dirac distribution. Unfortunately, no solution was found permitting the estimation of this distribution in the most general case. Indeed, we have to approximate $\epsilon(X)$ by its development at the second order in the coefficients of the vector $X$. In other words, we assume that a perturbation regime has been reached, for which the nonconstant part of the function $\epsilon(X)$ becomes small, when averaging over the distribution of the beam line errors, in such a way that the moments of order greater than two can be neglected. Thus, we write:

$$
\epsilon(X)=\sqrt{\operatorname{det}\left(\Sigma_{0}\right)}\left[1+\frac{1}{2} \mathrm{~T} X \Gamma_{0}^{-1} X\right]+\circ\left(X^{4}\right)
$$

where $\Gamma_{0}$ is the following $2 \times 2$ block diagonal matrix:

$$
\Gamma_{0} \stackrel{\text { def }}{=}\left(\begin{array}{cccc}
\Sigma_{0} & 0 & \ldots & 0 \\
0 & \Sigma_{0} & \ldots & 0 \\
\vdots & \vdots & \ddots & \vdots \\
0 & 0 & \ldots & \Sigma_{0}
\end{array}\right)
$$

Finally, with this approximation, we obtain [3]:

$$
\rho_{\epsilon}(\triangle \epsilon / \epsilon)=\frac{1}{2 \pi} \int_{-\infty}^{\infty} d k \exp (i k(\triangle \epsilon / \epsilon))\left(\prod_{p=1}^{2 n_{s}}\left(1+i k \lambda_{p}\right)\right)^{-\frac{1}{2}}
$$

where $\Delta \epsilon / \epsilon \stackrel{\text { def }}{=}\left(\epsilon-\sqrt{\operatorname{det} \Sigma_{0}}\right) / \sqrt{\operatorname{det} \Sigma_{0}}$ and where $\lambda_{p}$, $1 \leq p \leq 2 n_{s}$, are the $2 n_{s}$ eigenvalues of the matrix $\Gamma \Gamma_{0}^{-1}$.

\section{CONCLUSION}

Starting from a given probability law for the misalignments of the beam line components, a given trajectory correction scheme, and a given focusing lattice, the method computes the statistical distribution laws of certain quantities relative to the beam itself. Therefore, the power of this new approach lies in the fact that it can generate, in one single passage, statistical results which could be reproduced by a classical tracking program, but in a much more tedious way (several runs on several beam lines with randomly generated misalignments).

\section{REFERENCES}

[1] A. Chao, B. Richter, C.-Y. Yao, Nucl. Inst. Meth., 178 (1980) p. 1.

[2] T.O. Raubenheimer, K. Kubo, Nucl. Inst. Meth., A370 (1996) p. 303.

[3] S. Fartoukh. A Statistical Approach to Analyse the Efficiency of BNS Damping and Correction Algorithms in Linear Colliders. CERN/PS 97-06 (LP) (1997). 